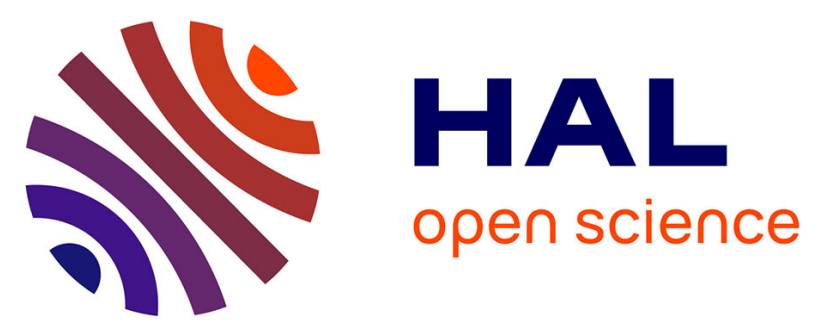

\title{
Tortuosity of mesoporous alumina catalyst supports: Influence of the pore network organization
}

\author{
Svetan Kolitcheff, Elsa Jolimaitre, Antoine Hugon, Jan Verstraete, \\ Pierre-Louis Carrette, Mélaz Tayakout-Fayolle
}

\section{- To cite this version:}

Svetan Kolitcheff, Elsa Jolimaitre, Antoine Hugon, Jan Verstraete, Pierre-Louis Carrette, et al.. Tortuosity of mesoporous alumina catalyst supports: Influence of the pore network organization. Microporous and Mesoporous Materials, 2017, 248, pp.91-98. 10.1016/j.micromeso.2017.04.010 . hal01581729

\section{HAL Id: hal-01581729 \\ https://hal-ifp.archives-ouvertes.fr/hal-01581729}

Submitted on 5 Sep 2017

HAL is a multi-disciplinary open access archive for the deposit and dissemination of scientific research documents, whether they are published or not. The documents may come from teaching and research institutions in France or abroad, or from public or private research centers.
L'archive ouverte pluridisciplinaire HAL, est destinée au dépôt et à la diffusion de documents scientifiques de niveau recherche, publiés ou non, émanant des établissements d'enseignement et de recherche français ou étrangers, des laboratoires publics ou privés. 


\title{
TORTUOSITY OF MESOPOROUS ALUMINA CATALYST SUPPORTS: INFLUENCE OF THE PORE NETWORK
} ORGANIZATION

\author{
Svetan Kolitcheff ${ }^{1}$, Elsa Jolimaitre ${ }^{1}$, Antoine Hugon ${ }^{1}$, Jan Verstraete ${ }^{1}$, Pierre-Louis Carrette ${ }^{1}$, Mélaz Tayakout- \\ Fayolle $^{2 *}$ \\ ${ }^{1}$ IFP Énergies nouvelles, Rond-point de l'échangeur de Solaize - BP 3 - 69360 Solaize, France \\ ${ }^{2}$ Laboratoire d'Automatique et de Génie des Procédés, UMR 5007, CNRS-ESCPE 43 Bd du 11 Novembre 1918, \\ 69622 Villeurbanne, France
}

* Corresponding author (tayakout@lagep.univ-lyon1.fr / Laboratoire d'Automatique et de Génie des Procédés, UMR 5007, CNRS-ESCPE 43 Bd du 11 Novembre 1918, 69622 Villeurbanne, France)

Abstract: Inverse liquid chromatography experiments were performed on five mesoporous alumina catalyst supports with similar porosity and different pore size distributions. By varying the size of the molecular tracer, it was shown that the diffusion regime in our conditions is molecular diffusion. Hindered diffusion was not observed even for squalane, a $\mathrm{C}_{30}$ molecule. Using the slope of the Van Deemter equation, the tortuosity of each alumina support was determined. The results are in disagreement with literature correlations: although all alumina supports had similar total porosities, the measured tortuosity values are really different and much higher than those predicted by these theoretical models. This discrepancy has been resolved by assuming a two-level pore network organization, whose characteristics can be entirely estimated from a classical nitrogen adsorption isotherm. This simple methodology allows to evaluate the mass transfer in mesoporous alumina supports knowing their textural properties, which is an important issue for the design and optimization of numerous catalytic processes.

Keywords: Alumina support; Inverse liquid chromatography; Internal diffusional limitations; Tortuosity; Pore network; Pore size distribution. 


\section{Introduction}

$\gamma$-Aluminas are used as catalyst supports in a variety of chemical and refinery processes. It is particularly the case in the field of petroleum (and recently biomass-derived oil) hydrotreating. Different support characteristics have a direct impact on the final activity of the catalyst, amongst which are the surface area and chemistry, the mechanical strength and the mass transfer properties. Over the past decades, a large amount of research has been dedicated to the optimization of the catalyst active phase (i.e. maximization of the selectivity and of the chemical kinetic rate), whereas mass transfer properties have been essentially put aside. As the particle size of industrial catalysts is generally chosen to be large in order to limit the bed pressure drop, mass transfer in the catalyst pore network may now become the limiting step for the new generation of industrial hydrotreating catalysts, in particular for heavy liquid petroleum fractions such as vacuum distillates or biomass-derived pyrolysis oils. It is therefore necessary to improve the characterization of mass transfer of liquids in mesoporous alumina supports in order to better understand the relationship between the synthesis conditions, the textural properties and the mass transfer kinetics.

Over the years, many experimental techniques have been implemented to measure mass transfer kinetics in porous solids. Given the constraint of liquid phase diffusion, Pulsed Field Gradient Nuclear Magnetic Resonance $(\mathrm{PFG}-\mathrm{NMR})^{[1,2]}$ and inverse chromatography ${ }^{[3]}$ can be considered as the most adequate. Because it is simple to operate and gives access to transport parameters (against self-diffusion coefficients for PFG-NMR), inverse chromatography was selected for this study. Recently, Soukup et al. ${ }^{[4]}$ evaluated diffusion coefficients in hydrotreating catalysts and supports by inverse chromatography. However, the experiments were operated in the gas phase and Knudsen diffusion was the predominant transport mechanism. Hence, the texture effects (for example the influence of the pore size distribution) cannot be extrapolated to the liquid phase. To the best of our knowledge, Inverse Liquid Chromatography (ILC) to study mesoporous alumina supports has never been reported in the literature so far. Nevertheless, the use of ILC to characterize transport properties of large molecule in silica has already been reported $^{[5]}$.

Two major difficulties arise when studying mass transfer of high molecular weight hydrocarbons inside mesoporous alumina supports.

First of all, the porous structures of industrial alumina supports strongly depend on their synthesis conditions, are highly complex and still not well characterized. An alumina catalyst support ( $\approx 1 \mathrm{~mm}$ of diameter) is essentially the result of the stacking of millions of elementary alumina nanocrystals $\left(\approx 10 \mathrm{~nm}\right.$ of diameter ${ }^{[6]}$. Given their tiny size, and their tendency to aggregate, the exact morphology of the nanocrystals and of the porous volume created in their vicinity is generally not accessible by microscopic techniques. The pore volume is therefore mostly characterized by mercury porosimetry and nitrogen adsorption analysis, yielding pore volumes and pore size distributions. Using 
different techniques, a recent study postulated that alumina catalyst supports are constituted of different scales of porosity $^{[2]}$.

The second difficulty is related to the diffusion mechanism, which in liquid phase can be either molecular diffusion (when intermolecular collisions are dominant, i.e. when the size of the molecule is small compared to that of the pore) or hindered diffusion (sometimes called surface diffusion, when the interactions between the molecule and the surface become predominant). For high molecular weight hydrocarbons in mesoporous pores, it is not clear which diffusion regime is rate-limiting, and this information has to be determined experimentally.

In the molecular diffusion regime, the conventional approach to account for the effect of the porous network is to introduce a correction of to the molecular diffusion coefficient $D_{m}$ by two textural parameters, the particle porosity $\varepsilon_{p}$ and the tortuosity $\tau$ in order to obtain the well-known effective diffusion coefficient: $D_{\text {eff }}=D_{m} \cdot \varepsilon_{p} / \tau(1)$

The porosity represents the void fraction inside the porous particles and can easily be evaluated from classical porosimetry techniques. Evaluation of the tortuosity of a given porous system is, however, much more complicated. From a geometric point of view, the tortuosity represents the ratio between the total length of the diffusion path of the fluid in a porous medium and the corresponding straight-line distance. From a macroscopic point of view, equation 1 links two diffusion coefficients, with and without the presence of the porous medium, and defines the tortuosity as a correction factor that is necessary to account for the presence of the porous medium once the void fraction has been taken into account. The tortuosity factor therefore depends on the support porosity (i.e. the tortuosity decreases, if the void fraction increases) and the network structure, but, in the molecular diffusion regime, it does not depend on the size of the molecular tracer. Indeed, if molecules of strongly differing sizes are not able to follow the same paths in the porous network, the diffusion mechanism is surface hindered.

The simplest way to evaluate $\tau$ is to apply one of the numerous of theoretical or empirical relations between $\tau$ and $\varepsilon_{p}$ that have been proposed in literature ${ }^{[7,8]}$. Unfortunately, to select an appropriate relation, a good knowledge of the geometric properties of the system (shape, size and size distribution of the elementary nanocrystals) is required, which is rarely available for most alumina supports. Moreover, "real" solids often differ significantly from ideal stacking systems considered in theoretical works. It is therefore often necessary to evaluate $\tau$ experimentally. To do so, the best solution is to measure the effective diffusion and use equation (1) to calculate the tortuosity factor.

In this work, five mesoporous $\gamma$-alumina supports of equal porosity and different pore size distributions were studied. To characterize their diffusional properties, inverse liquid chromatography (ILC) experiments were performed with two molecules of strongly different sizes: methylcyclohexane $\left(\mathrm{C}_{7} \mathrm{H}_{14}\right)$ and squalane $\left(\mathrm{C}_{30} \mathrm{H}_{62}\right)$. Both tracers were diluted in $n$-heptane to determine both the diffusion regime and the tortuosity values. Finally, a decomposition of the porosity into two different porous networks is proposed to explain the diffusion behavior inside the studied materials. 


\section{Experimental}

\subsection{Materials and texture analysis}

Five boehmite $\gamma$-alumina supports, provided by IFPEN, were studied in this work. The $\gamma$-alumina supports are obtained by precipitation of aluminum salts in an aqueous solution. The boehmite precipitate was filtered and washed. Shaping involves the passage from a boehmite powder to support pellets. The extrudates are trilobal. Their diameter ranges from 1.2 to $2 \mathrm{~mm}$ and the lengths from 2 to $6 \mathrm{~mm}$. A thermal treatment at high temperature (from 798 to $1248 \mathrm{~K}$ ) was performed to obtain the final support. The aim of these thermal treatments is to optimize the particle size, which increases with temperature, the average pore diameter, the total pore volume, and the surface area. The final $\gamma$-alumina support has a purity $>99 \%$ by weight. The added impurities, introduced during the precipitation in the aqueous solution, are $\mathrm{P}, \mathrm{Na}, \mathrm{Cl}$ and $\mathrm{Mg}$ elements. These impurity quantities have not effect on textural properties.

Textural properties were measured by physical nitrogen adsorption on an ASAP 2420 instrument and helium pycnometry on an Accupyc 1340 instrument. The BET surface area $S_{B E T}$, the pore volume $V_{p}$ and the microporous volume were evaluated form the nitrogen isotherm. Helium pycnometry provided the structural density $\rho_{s}$. The porosity $\varepsilon_{p}$ of all the studied solids was determined according to the following expression: $\varepsilon_{p}=\frac{V_{p}}{V_{p}+\frac{1}{\rho_{s}}}$ (2). The textural properties of all alumina supports are reported in Table I. All studied alumina supports are strictly mesoporous. As the alumina supports were selected to have very similar porosities in order to focus on the tortuosity effect, the porous volume and structural density are very close for all samples. Only the BET surface varies significantly, meaning that the nanocrystals used for the alumina support synthesis display different surface/volume ratios. The BJH method was used to estimate the pore size distributions of the studied alumina supports reported in Figure 1 from the nitrogen desorption branch. The pore size distributions of the different samples vary significantly. Alumina supports B and D seem to be almost mono-disperse, while the three others are at least bimodal. Besides, the maximum of the curve is around $5 \mathrm{~nm}$ for sample A and nearly $17 \mathrm{~nm}$ for sample E.

\subsection{Inverse liquid chromatography}

Inverse liquid chromatography (ILC) was used to characterize the diffusion properties in alumina supports. The ILC setup is presented in Figure 2. 
Stainless columns of $50 \mathrm{~cm}$ in length and $1 \mathrm{~cm}$ in internal diameter were filled with crushed and sieved alumina supports. The mean alumina support radii $R_{p}$ were measured by laser granulometry with a Matersizer 3000 . All samples were activated under nitrogen $\left(1 \mathrm{NL} \cdot \mathrm{h}^{-1}\right)$ at $350{ }^{\circ} \mathrm{C}$ with a $5{ }^{\circ} \mathrm{C} \cdot \mathrm{min}^{-1}$ ramp for $12 \mathrm{~h}$ prior to measurement.

A probe molecule diluted in a solvent was used as a tracer. The solvent and the tracers were chosen to satisfy the following criteria: they needed to be miscible, have a low adsorption coefficient on aluminas, have different refractive indices, and the tracers needed to have different molecular sizes. Binary solutions containing 3 wt $\%$ of methylcyclohexane $(\mathrm{MCH})$ or squalane (SQ) as tracer in $n$-heptane (C7) solvent were used, and respectively designated as MCH-C7 and SQ-C7.

The inverse chromatography experiments were performed at $35{ }^{\circ} \mathrm{C}$, starting with columns that were initially filled with the solvent. Liquid flow rates were varied from 1 to $10 \mathrm{~cm}^{3}$ per minute. A 4-port valve connects either the solvent circuit or tracer solution circuit to the column. The tracer inlet concentration to the column was therefore a step function in the ILC experiments. The tracer outlet concentration was measured at a frequency of $1 \mathrm{~Hz}$ by an online UR24 High Accuracy refractometer with absolute refractive index precision of $0.00007 \mathrm{nD}$. After the system reached equilibrium (i.e. the tracer concentration at the outlet of the column has reached the inlet concentration), pure solvent is injected in the column. Hence, for each experiment, both the positive steps (breakthrough curves) and the negative steps (inverse breakthrough curves, also called purge curves) were performed. From the mass balances, the amount of adsorbed tracer and the explored porosity in the porous aluminas can be determined.

\subsection{Mass transfer modelling}

Transport parameters were evaluated using the first and second moments of the experimental responses ${ }^{[3]}$. For an inverse liquid chromatography experiment with a step function as input, the first moment $\mu_{1}$ is equal to the mean residence time of the tracer within the column, while the second moment characterizes the dispersion of the tracer. Instead of the second moment, the standard deviation or second central moment $\sigma^{2}\left(\mathrm{~s}^{2}\right)$ is usually preferred: $\sigma^{2}=$ 2. $\mu_{2}-\mu_{1}^{2}$ (3), where sigma represents the standard deviation.

The theoretical moments, containing the mass transfer parameters, depend on the column model that is used. For a mono-disperse linear model, the expression of the first moment does not depend on the mass transfer properties and is given by the following equation, for a mono-disperse linear model ${ }^{[9]}$ :

$$
\mu_{1}=\frac{L}{v_{i}}\left[1+\left(\frac{1-\varepsilon_{i}}{\varepsilon_{i}}\right) \cdot K\right](4)
$$

where $\mathrm{L}$ is the column length, $v_{i}$ the interstitial velocity of the fluid, $\varepsilon_{i}$ the interstitial or bed porosity and $K$ is referring to the following expression:

$$
K=\left(\varepsilon_{p}+\left(1-\varepsilon_{p}\right) \cdot K_{j}\right)(5)
$$


where $K_{j}$ is the local slope of the co-adsorption (SQ/C7 or $\left.\mathrm{MCH} / \mathrm{C} 7\right)$ isotherm, and $\varepsilon_{p}$ the porosity of the porous support.

The second central moment is defined by:

$$
\sigma^{2}=2 \cdot\left(\frac{L}{v_{i}}\right) \cdot\left(\frac{D_{L}}{v_{i}^{2}}\right) \cdot\left(1+\left(\frac{1-\varepsilon_{i}}{\varepsilon_{i}}\right) \cdot K\right)^{2}+\frac{2}{3} \cdot\left(\frac{L}{v_{i}}\right) \cdot\left(\frac{R_{p}}{k_{f}}+\frac{R_{p}^{2}}{5 \cdot D_{e f f}}\right) \cdot\left(\frac{1-\varepsilon_{i}}{\varepsilon_{i}}\right) \cdot K^{2}(6)
$$

where $D_{L}$ is the axial dispersion coefficient in the packed bed, $k_{f}$ the external fluid film mass transfer coefficient, and $D_{\text {eff }}$ the effective diffusion coefficient inside the porous particles.

The evolution of the experimental moments with the interstitial velocity $v_{i}$ is commonly studied via the Height Equivalent to a Theoretical Plate, HETP, and is defined as follows: HETP $=\frac{\sigma^{2}}{\mu_{1}^{2}} \cdot L(7)$. This concept is analogous to the approach by continuous stirred tank reactors (CSTRs) in series of a of a non-ideal flow reactor. For a monodisperse model:

$$
\begin{aligned}
& \text { HETP }=2 \cdot \frac{D_{L}}{v_{i}}+v_{i} \cdot\left[\frac{R_{p}}{k_{f}}+\frac{1}{5} \cdot \frac{R_{p}{ }^{2}}{D_{e f f}}\right] \cdot X(8) \\
& \text { with } X=\frac{2}{3} \cdot \frac{\left(\frac{1-\varepsilon_{i}}{\varepsilon_{i}}\right) \cdot K^{2}}{\left(1+\left(\frac{1-\varepsilon_{i}}{\varepsilon_{i}}\right) \cdot K\right)^{2}}(9)
\end{aligned}
$$

where $X$ is the column factor, $R_{p} / k_{f}$ is the external diffusion characteristic time and $R_{p}{ }^{2} / D_{\text {eff }}$ is the internal diffusion characteristic time. The column factor is used to take into account the interstitial porosity within different columns. If the main contribution to the mass transfer limitation is external diffusion, equation (8) becomes: HETP = $2 \cdot \frac{D_{L}}{v_{i}}+v_{i} \cdot \frac{R_{p}}{k_{f}} \cdot X(10)$. On the other hand, if internal diffusion is limiting, equation (8) becomes: HETP $=2 \cdot \frac{D_{L}}{v_{i}}+$ $v_{i} \cdot \frac{1}{5} \cdot \frac{R_{p}^{2}}{D_{e f f}} \cdot X(11)$

Axial dispersion $D_{L}$ in the packed bed is due to two main mechanisms: molecular diffusion and turbulent mixing. Considering that these effects are additive gives: $D_{L}=\gamma_{1} \cdot D_{m}+2 \cdot \gamma_{2} \cdot R_{p} \cdot v_{i}(12)$ where $\gamma_{1}$ and $\gamma_{2}$ are constants.

Combining equations (8) and (12) yields a relation that takes the same general form as the van Deemter equation: HETP $=A_{1}+\frac{A_{2}}{v_{i}}+A_{3} \cdot v_{i}(13)$. In this equation, the constant term $A_{1}$ depends on the particle size, term $A_{2}$ depends on the molecular diffusion coefficient, while term $A_{3}$ depends on particle size, external fluid film mass transfer, effective diffusion, adsorption, interstitial porosity, and particle porosity.

In the liquid phase, the term $\gamma_{1} \cdot D_{m}$ is negligible compared to $2 \cdot \gamma_{2} \cdot R_{p} \cdot v_{i}$, and parameter $A_{2}$ is generally negligible ${ }^{[10]}$. The HETP should therefore increase linearly with $v_{i}$, and its slope $A_{3}$ is directly related to mass transfer and adsorption properties. When adsorption can be neglected (which is the case for our systems, as will be shown in section 3.1.), equation (5) becomes: $K=\varepsilon_{p}$ (14). 


\section{Results}

\subsection{Internal diffusional limitation}

As shown by equations (10) and (11), the radius of the alumina support particles does not have the same impact on internal and external (film) diffusion limitations. It is therefore possible to separate external and internal mass transfer contributions by performing experiments with alumina supports of different sizes. ILC experiments with SQC7 were performed on alumina D particles of different sizes. Two columns, indexed 1 and 2 , were filled with alumina support particles with a mean radius of 0.303 and $0.422 \mathrm{~mm}$ respectively. The evolutions of the HETP as the function of interstitial velocity are reported for both columns in Figure 3a. As expected, the experimentally measured HETPs vary linearly with interstitial velocity, which allows to determine the mass transfer limitation properties. The linear regressions of these curves provide the characteristic time for diffusion, $A_{3}$. Table II reports, for both columns, the ratio of the slopes and the single and squared ratios of alumina support radii corrected by the column factors $X_{\mathrm{i}}$. The value of ratio of the slopes is equal to the ratio of the squared particle radii, demonstrating that the resistance from the external diffusion is negligible in the overall mass transfer. Equation (8) therefore becomes equation (11).

\subsection{Molecular diffusion regime}

To identify the influence of the tracer size on the transport property in alumina support, ILC measurements for MCH-C7 and SQ-C7 solutions were performed on alumina support D. In the experiments, the first moment (retention time) is the same for both tracers, but the second moments are different. For all aluminas, the explored porosity, both with methyl cyclohexane and squalane as tracer molecules, was also in agreement with the helium and nitrogen measurements. Moreover, the competitive adsorption was found to be negligible. In the studied conditions, the breakthrough curves and the inverse breakthrough curves (as defined in section 2.2) could not be distinguished, since neither the solvent nor tracers adsorb. Hence, both curves provide identical information and can be used interchangeably to estimate the slope of the HETP curve, as the same values for the first and second moment were obtained.

Figure $3 \mathrm{~b}$ shows the evolution of the HETP with the interstitial velocity for both tracers. If the diffusion regime is molecular, the tortuosity should be the same for both molecules and correspond to the geometrical tortuosity of the porous network. If hindered diffusion is not negligible, the effective diffusion should be smaller for the bigger molecule (SQ) and if the porosity remains constant, the corresponding tortuosity increases. Effective diffusion coefficients $D_{\text {eff }}$ estimated from the curves slopes are given in Table III. By repeating several breakthrough curves 
on various columns with the same solid, it was shown that the effective diffusion coefficients can be determined in our system with a relative error of $5 \%$.

In order to calculate the tortuosity, molecular diffusion coefficients for both solutions have been estimated from the Hayduk and Minhas correlation ${ }^{[11]}$. The molecular diffusion coefficients and tortuosity values (calculated from equation (1)) for both tracers are given in Table III for alumina support D. The tortuosity values are the same for MCH-C7 and SQ-C7. Since the tortuosity does not vary with molecular size, hindered diffusion is negligible. As shown in Table IV, the same result is obtained for all samples. The molecular diffusion regime is therefore confirmed for both tracers in all alumina supports, even for squalane which is a $\mathrm{C}_{30} \mathrm{H}_{62}$ aliphatic compound.

\section{Discussion}

\subsection{Correlations with the porous network}

Mesoporous alumina supports with similar porosity but different pore size distributions and specific surfaces have been characterized by ILC. The resulting tortuosity values, reported in Table IV, vary between 2 and 3 depending on the sample. As shown in the previous section, there is strong evidence that the diffusion regime is molecular and hence, neither the specific surface nor the pore size distribution should affect the effective diffusivities.

Behind those textural properties, the organization of the porous network may play a role in the diffusion behavior inside the solids. To investigate the pore network organization, the measured tortuosity values were compared to theoretical relations between tortuosity and porosity. Such relations are based on models for the structure and organization of a porous medium ${ }^{[7,8]}$. Table $\mathrm{V}$ reports some of these theoretical tortuosity-porosity relations for several pore structure models, along with the physical hypotheses on which they were elaborated. Curves corresponding to those relations and our measured tortuosity values are shown in Figure 4.

With an overall porosity of around 0.7 , the measured tortuosity values of our solids (between 2 and 3) are much higher than the tortuosity values predicted by the correlations (between 1.2 and 1.4), suggesting that only part of the porosity of the alumina supports actively contributes to the mass transfer.

\subsection{Hierarchized porous material}

To take into account that all the pore volume is not equivalent as far as its contribution to the mass transfer resistance is concerned, an organization of the porous network in different levels was assumed. As suggested by recent studies ${ }^{[2]}$, this organization could be constituted of two levels, as illustrated in Figure 5. Microscopically, alumina nanocrystals are combined to form aggregates. A first level of porosity can therefore be created between 
these alumina nanocrystals inside the aggregates. These alumina aggregates will then be compressed together to create the catalyst support. Hence, at a larger scale, a second level of porosity exists in between the aggregates. In this work, the alumina support will be represented by alumina aggregates that are composed of smaller alumina nanocrystals, thereby creating two levels of porosity, an internal pore network inside the aggregates (porosity 1, and noted $\varepsilon_{p 1}$ ) and external pore network around the aggregates (porosity 2, and noted $\varepsilon_{p 2}$ ).

Looking at the mass transfer inside the alumina support at both levels, one can now distinguish two characteristic diffusion times:

- The first level, called the aggregates, results from the stacking of the elementary nanocrystals of alumina. For this stacking to be sufficiently stable (i.e. densely packed), the size of the interstices between the nanocrystals should be close to the diameter of the nanocrystals ${ }^{[12]}$. The characteristic diffusion time for this first level is therefore: $t_{1}=\frac{R_{a g g}{ }^{2}}{5 \cdot \frac{\varepsilon_{p 1}}{\tau_{1}} \cdot D_{m}}(15)$

- The second level concerns the porosity around the aggregates, which constitutes a second pore network. In order to diffuse throughout the alumina support, the molecules have to use this network. Hence, the characteristic diffusion time for this second level is therefore: $t_{2}=\frac{R_{p}^{2}}{5 \cdot \frac{\varepsilon_{p 2}^{2}}{\tau_{2}} \cdot D_{m}}(16)$

Assuming that the radius of the aggregates $R_{a g g}$ is much smaller than the radius $R_{p}$ of the alumina supports yields $t_{1} \ll t_{2}$, i.e. the second level constitutes the only diffusion limiting level in the alumina support.

This organization in two levels of porosity, characterized by two mass transfer resistances in series, is commonly encountered for solid catalysts or adsorbents synthesized by the extrusion of micrometric powders. In our case, one of the resistances (that associated to the first level, i.e. the internal pore network inside the aggregates) is considered as negligible.

In order to evaluate the volumes corresponding to these different porous networks, the BJH pore size distributions are decomposed.

The main assumptions of the decomposition method are listed below.

- $\quad$ Besides the bed porosity $\varepsilon_{i}$, two levels of porosity in the alumina supports are considered, one associated to the porosity inside the aggregates - called porosity 1 and noted $\varepsilon_{p 1}$ - and the other associated the porosity between the aggregates - called porosity 2 and noted $\varepsilon_{p 2}$.

- $\quad$ The pore size distribution for each level is supposed to follow a normal distribution: $x V_{\mathrm{i}}(d)=\frac{x V_{\mathrm{i}}^{T}}{\sigma_{i} \cdot \sqrt{2 \pi}}$. $\exp \left(-\frac{1}{2} \cdot\left(\frac{d-d_{i}}{\sigma_{\mathrm{i}}}\right)^{2}(17)\right.$

where $d$ is the pore diameter, while $\mathrm{d}_{\mathrm{i}}, V_{\mathrm{i}}^{T}$ and $\sigma_{\mathrm{i}}$ are respectively the mean pore size, the volume fraction and the standard deviation of the mean pore size associated to each level of porosity. 
- The alumina nanocrystals are platelets, as shown in Figure 5a. In order to calculate a characteristic length, they will be approximated as spheres, and their diameter can therefore be estimated from the experimental $D_{\text {nanocrystals }}=6 /\left(S_{B E T} \cdot \rho_{\mathrm{s}}\right)(18)$

- The average pore diameter $d_{1}$ of porosity 1 is assumed to be equivalent to that of the nanocrystals $\left(d_{1}=\right.$ $\left.D_{\text {nanocrystals }}(19)\right)$. As mentioned above, this relation is generally found in the case of dense stacking of spherical alumina supports ${ }^{[12]}$. The values of the average porous diameter for the first level of porosity $\left(\mathrm{d}_{1}\right)$ for each alumina support are reported in Table VI.

All the other parameters $\left(d_{2}(\mathrm{~nm}), \sigma_{1}(\mathrm{~nm}), \sigma_{2}(\mathrm{~nm}), V_{1}^{T}, V_{2}^{T}\right)$ have been estimated by fitting the sum of the two normal distributions with the experimental pore size distribution. Figure 6 shows a good agreement between the simulated and experimental alumina pore size distributions. The corresponding estimated parameters are given Table VI. As expected, the average pore diameters $\left(d_{2}\right)$ corresponding to porosity 2 are larger than those $\left(d_{1}\right)$ obtained for porosity 1 . The proportion of the porosity $\left(V_{2}^{T}\right)$ increases with the average pore diameter. From this parameter, the porosity around the aggregates has been calculated as follows $\varepsilon_{p 2}=V_{2}^{T} \cdot \varepsilon_{\mathrm{p}}(20)$ and reported in Table VI. Since the mass transfer resistance of the alumina support is located only in the second porosity, the two characteristic diffusion times are equivalent and the tortuosity associated to porosity 2 can be calculated as follows: $\tau_{2}=\varepsilon_{p 2} \cdot \frac{\tau}{\varepsilon_{\mathrm{p}}}(21)$. The resulting values are given in Table VI and compared with the theoretical correlations in Figure 7.

The variation of $\tau_{2}$ with $\varepsilon_{p 2}$ is clearly in better agreement with literature correlations than the variations of $\tau$ with $\varepsilon_{\mathrm{p}}$. The tortuosity of the second level of porosity is consistent with the geometric tortuosity of a system of overlapping cylinder, with values varying between 1.82 and 1.56 for tortuosity values for porosity between 0.44 and 0.57 . The inconsistency between certain values (for example the tortuosity of sample B is higher than that of A, whereas the porosity of the latter is smaller) can probably be attributed to experimental uncertainties.

This analysis shows that, in order to optimize mass transfer in the aluminas support, the volume between the aggregates should be maximized. In other words, synthesis conditions have to be adapted in order to minimize the aggregation of the nanocrystals, allowing all the porous volume to contribute efficiently to diffusion. A question still pending is the pertinence of this analysis for actual catalysts, that is to say alumina supports impregnated with an active phase, which can account for more than $20 \%$ of the mass of the solid. The effects could be very different depending on the location of this active phase: in the intra-aggregate volume, mass transfer should hardly be impacted, whereas in the inter-aggregate volume, mass transfer could be significantly slowed down.

\section{Conclusion}

Mass transfer properties, more specifically tortuosity values, were determined by inverse liquid chromatography for five mesoporous alumina supports, representative of petroleum hydrotreating catalyst supports. In the 
experimental conditions studied (liquid phase at $35^{\circ} \mathrm{C}$ ), the hypothesis of the molecular diffusion regime was confirmed both for small (heptane and methylcyclohexane) and larger (squalane, a $\mathrm{C}_{30} \mathrm{H}_{62}$ ) molecules.

Although all aluminas had similar porosities, their tortuosity values were quite different. The estimated tortuosity values disagree with literature correlations on two points: the values are higher and vary much more with porosity than predicted. This result was explained by the presence of a hierarchized organization inside the alumina support: the alumina nanocrystals are first stacked into aggregates (resulting in a first level of porosity), which gather together (creating a second level of porosity) to form the final alumina support. The second level of porosity, which permeates throughout the alumina support, is expected to be the limiting porous network for mass transfer.

Using the specific surface of the solids, the pore size distribution obtained by the BJH method was decomposed, yielding the porous volumes associated to the two levels. The parameters for this two-level porosity model were calculated for the five aluminas. The results showed that a higher mean pore diameter for the porosity inside the aggregates leads to a higher fraction of the pore volume outside the alumina aggregates. Finally, the intrinsic tortuosity of the second level was evaluated, and successfully compared to the literature correlations.

The proposed two-level porosity representation method allows to explain how aluminas with a high porosity $\left(\varepsilon_{p} \approx 0.7\right)$ are able to exhibit high tortuosity values $(\tau \approx 2-3)$ despite the low solid fraction in the particles. From this analysis, we can conclude that mass transfer in alumina supports can be optimized by avoiding the agglomeration of the nanocrystals during their synthesis. In order to extend this conclusion to actual hydrotreating catalysts, the effect of the active phase deposition will have to be evaluated.

Ultimately, the methodology proposed in this work enables to extract mass transfer properties from simple nitrogen adsorption experiments and, in that, it can be useful for the heterogeneous catalyst community.

\section{Symbols}

$\begin{array}{lll}C_{0} & \text { mol.m } & \text { Tracer initial concentration } \\ C(t) & \text { mol.m } & \text { Tracer concentration over time } \\ d & \mathrm{~nm} & \text { Porous diameter } \\ d_{1} & \mathrm{~nm} & \text { Mean pore size of the porosity } 1 \\ d_{2} & \mathrm{~nm} & \text { Mean pore size of the porosity } 2 \\ D_{e f f} & \mathrm{~m}^{2} \cdot \mathrm{s}^{-1} & \text { Effective diffusivity } \\ D_{L} & \mathrm{~m}^{2} \cdot \mathrm{s}^{-1} & \text { Axial dispersion coefficient } \\ D_{m} & \mathrm{~m}^{2} \cdot \mathrm{s}^{-1} & \text { Molecular diffusivity } \\ \varepsilon_{i} & - & \text { Interstitial porosity }\end{array}$




\begin{tabular}{|c|c|c|}
\hline$\varepsilon_{p}$ & - & Material porosity \\
\hline$\varepsilon_{p 1}$ & - & Porosity of the porosity 1 \\
\hline$\varepsilon_{p 2}$ & - & Porosity of the porosity 2 \\
\hline$\gamma_{1}, \gamma_{2}$ & - & Constants in equation 12 \\
\hline HETP & $\mathrm{cm}$ & Height Equivalent of a Theoretical Plate \\
\hline$k_{f}$ & $\mathrm{~m} \cdot \mathrm{s}^{-1}$ & External fluid film mass transfer coefficient \\
\hline$K_{j}$ & - & Henry law constant \\
\hline$L$ & $\mathrm{~m}$ & Column length \\
\hline$\mu_{1}$ & s & First order moment \\
\hline$\mu_{n}$ & - & $\mathrm{n}^{\text {th }}$ order moment \\
\hline$R_{p}$ & $\mathrm{~m}$ & Mean alumina supports radius \\
\hline$R_{\text {agg }}$ & $\mathrm{m}$ & Mean aggregates radius \\
\hline$\rho_{s}$ & g.cm $\mathrm{cm}^{-3}$ & Structural density measured by helium pycnometry \\
\hline$\sigma^{2}$ & $s^{2}$ & Centered second moment \\
\hline$\sigma_{1}$ & $\mathrm{~nm}$ & Standard deviation of the mean pore size in the porosity 1 \\
\hline$\sigma_{2}$ & $\mathrm{~nm}$ & Standard deviation of the mean pore size in the porosity 2 \\
\hline$S_{B E T}$ & $\mathrm{~m}^{2} \cdot \mathrm{g}^{-1}$ & BET surface \\
\hline$t$ & $\mathrm{~s}$ & Time \\
\hline$t_{1}$ & $\mathrm{~s}$ & Intra-aggregate characteristic diffusion time \\
\hline$t_{2}$ & s & Inter-aggregate characteristic diffusion time \\
\hline$\tau$ & - & Tortuosity \\
\hline$\tau_{1}$ & - & Tortuosity of the porosity 1 \\
\hline$\tau_{2}$ & - & Tortuosity of the porosity 2 \\
\hline$v_{i}$ & $\mathrm{~m} \cdot \mathrm{s}^{-1}$ & Interstitial velocity of fluid \\
\hline$V_{p}$ & $\mathrm{~cm}^{3} \cdot \mathrm{g}^{-1}$ & Porous volume per solid quantity \\
\hline$x V_{1}^{T}$ & $\mathrm{~cm}^{3} \cdot \mathrm{cm}^{-3}$ & Part of the overall porosity include in the porosity 1 \\
\hline$x V_{2}^{T}$ & $\mathrm{~cm}^{3} \cdot \mathrm{cm}^{-3}$ & Part of the overall porosity include in the porosity 2 \\
\hline$X$ & - & Column factor: $X=\frac{2}{3} \cdot \frac{\left(\frac{1-\varepsilon_{i}}{\varepsilon_{i}}\right) \cdot K^{2}}{\left(1+\left(\frac{1-\varepsilon_{i}}{\varepsilon_{i}}\right) \cdot K\right)^{2}}$ \\
\hline
\end{tabular}




\section{Acknowledgements}

\section{References}

[1] U. Tallarek, F. J. Vergeldt, H. Van As, 1999, 7654-7664.

[2] E. M. Forman, M. A. Trujillo, K. J. Ziegler, S. A. Bradley, H. Wang, S. Prabhakar, S. Vasenkov, Microporous Mesoporous Mater. 2016, 229, 117-123.

[3] D. M. Ruthven, Principles of Adsorption and Adsorption Processes, 1984.

[4] K. Soukup, M. Procházka, 2015, 43, 841-846.

[5] V. Wernert, R. Bouchet, R. Denoyel, Anal. Chem. 2010, 82, 2668-2679.

[6] D. Chiche, M. Digne, R. Revel, C. Chaneac, J. P. Jolivet, J. Phys. Chem. C 2008, $112,8524-8533$.

[7] L. Shen, Z. Chen, Chem. Eng. Sci. 2007, 62, 3748-3755.

[8] N. Wakao, J. M. Smith, Chem. Eng. Sci. 1962, 17, 825-834.

[9] H. W. Haynes, P. N. Sarma, Am. Inst. Chem. Eng. 1973, 19, 1043-1046.

[10] J. Kärger, D. M. Ruthven, D. N. Theodorou, Diffusion in Nanoporous Materials, 2012.

[11] W. Hayduk, B. S. Minhas, Can. J. Chem. Eng. 1982, 295-299.

[12] G. T. Nolan, P. E. Kavanagh, Powder Technol. 1994, 78, 231-238.

[13] K. A. Akanni, J. W. Evans, Chem. Eng. Sci. 1987, 42, 1945-1954.

[14] E. E. Petersen, Am. Inst. Chem. Eng. J. 1958, 4, 343-345.

[15] H. L. Weissberg, J. Appl. Phys. 1963, 34, 2636-2639. 
[16] M. M. Tomadakis, S. V Sotirchos, J. Chem. Phys. 1993, 98, 616-626.

\section{Tables}

Table I

Alumina supports textural properties

\begin{tabular}{ccccc}
\hline Alumina support & $S_{B E T}\left(\mathrm{~m}^{2} \cdot \mathrm{g}^{-1}\right)$ & $V_{p}\left(\mathrm{~cm}^{3} \cdot \mathrm{g}^{-1}\right)$ & $\rho_{s}\left(\mathrm{~g} \cdot \mathrm{cm}^{-3}\right)$ & $\varepsilon_{p}$ \\
\hline A & 340 & 0.71 & 3.3 & 0.72 \\
B & 300 & 0.72 & 3.3 & 0.70 \\
C & 290 & 0.78 & 3.3 & 0.72 \\
D & 270 & 0.74 & 3.3 & 0.71 \\
E & 160 & 0.77 & 3.5 & 0.73 \\
\hline
\end{tabular}

\section{Table II}

Mass transfer contributions

\begin{tabular}{|c|c|c|}
\hline$\frac{A_{3,1}}{A_{3,2}}$ & $\frac{R_{p, 1}}{R_{p, 2}} \cdot \frac{X_{1}}{X_{2}}$ & $\left(\frac{R_{p, 1}}{R_{p, 2}}\right)^{2} \cdot \frac{X_{1}}{X_{2}}$ \\
\hline 0.52 & 0.71 & 0.51 \\
\hline
\end{tabular}

\section{Table III}

Tortuosity values estimated for both tracers in alumina support A

\begin{tabular}{rcccc}
\hline Tracer & $A_{3}\left(\mathrm{~s}^{-1}\right)$ & $D_{\text {eff }}\left(10^{-9} \mathrm{~m}^{2} \cdot \mathrm{s}^{-1}\right)$ & $D_{m}\left(10^{-9} \mathrm{~m}^{2} \cdot \mathrm{s}^{-1}\right)$ & $\tau$ \\
\hline MCH-C7 & 3.50 & 1.2 & 4.1 & 2.4 \\
SQ-C7 & 9.10 & 0.46 & 1.6 & 2.5 \\
\hline
\end{tabular}




\section{Table IV}

Tortuosity values for all alumina supports

\begin{tabular}{ccc}
\hline & \multicolumn{2}{c}{$\tau$} \\
\hline Alumina support & MCH-C7 & SQ-C7 \\
\hline A & 3.0 & 3.0 \\
B & 2.8 & 2.8 \\
C & 2.4 & 2.4 \\
D & 2.4 & 2.5 \\
E & 2.0 & 2.0 \\
\hline
\end{tabular}

\section{Table V}

Physical systems, theoretical tortuosity-porosity relations and references

\begin{tabular}{lcc}
\hline \multicolumn{1}{c}{ Remarks } & Relations & References \\
\hline Random homogeneous isotropic sphere packings & $\tau=\frac{3-\varepsilon_{\mathrm{p}}}{2}$ & \\
Infinite cylinder & $\tau=2-\varepsilon_{\mathrm{p}}$ \\
Overlapping spheres & $\tau=1-\frac{1}{2} \ln \varepsilon_{\mathrm{p}}$ \\
Overlapping cylinder & $\tau=1-\ln \varepsilon_{\mathrm{p}}$ \\
Cuboid & $\tau=\frac{1}{\varepsilon_{\mathrm{p}}}$
\end{tabular}

Table VI

Estimated parameters for the pore size distribution decomposition and resulting porosity and tortuosity for the limiting porous network.

\begin{tabular}{ccccccc|cc}
\hline & $d_{1}(\mathrm{~nm})$ & $d_{2}(\mathrm{~nm})$ & $\sigma_{1}(\mathrm{~nm})$ & $\sigma_{2}(\mathrm{~nm})$ & $x V_{1}^{T}$ & $x V_{2}^{T}$ & $\varepsilon_{p 2}$ & $\tau_{2}$ \\
\hline A & 5.4 & 8.0 & 1.2 & 2.6 & 0.38 & 0.62 & 0.44 & 1.9 \\
B & 6.0 & 8.0 & 1.6 & 0.94 & 0.30 & 0.70 & 0.49 & 1.9 \\
C & 6.4 & 9.6 & 1.1 & 2.7 & 0.26 & 0.74 & 0.53 & 1.8 \\
D & 6.8 & 9.5 & 1.3 & 1.1 & 0.29 & 0.71 & 0.50 & 1.8 \\
E & 11 & 17 & 2.1 & 3.2 & 0.21 & 0.79 & 0.57 & 1.6 \\
\hline
\end{tabular}




\section{Figure captions}

Figure 1. Alumina supports pore size distribution estimated by the BJH method

Figure 2. Inverse liquid chromatography setup

Figure 3. van Deemter curves for alumina support D: (a) effect of particle size with SQ-C7 and (b) effect of the tracer size (MCH-C7 vs. SQ-C7)

Figure 4. Theoretical and experimental (ILC) tortuosity-porosity values

Figure 5. (a) Schematic representation of a typical alumina platelet shape ${ }^{[6]}$ and (b) Schematic representation of the two levels of porosity

Figure 6. Deconvolution of the BJH pore size distributions for the various alumina supports into two pore size distributions, one for the porosity intra-aggregate and one for the porosity inter-aggregate.

Figure 7. Theoretical and experimental (Inverse Liquid Chromatography) tortuosity-porosity relations. ILC corresponds to the uniform porosity representation - revised ILC corresponds to the two-level porosity representation.

\section{Figures}

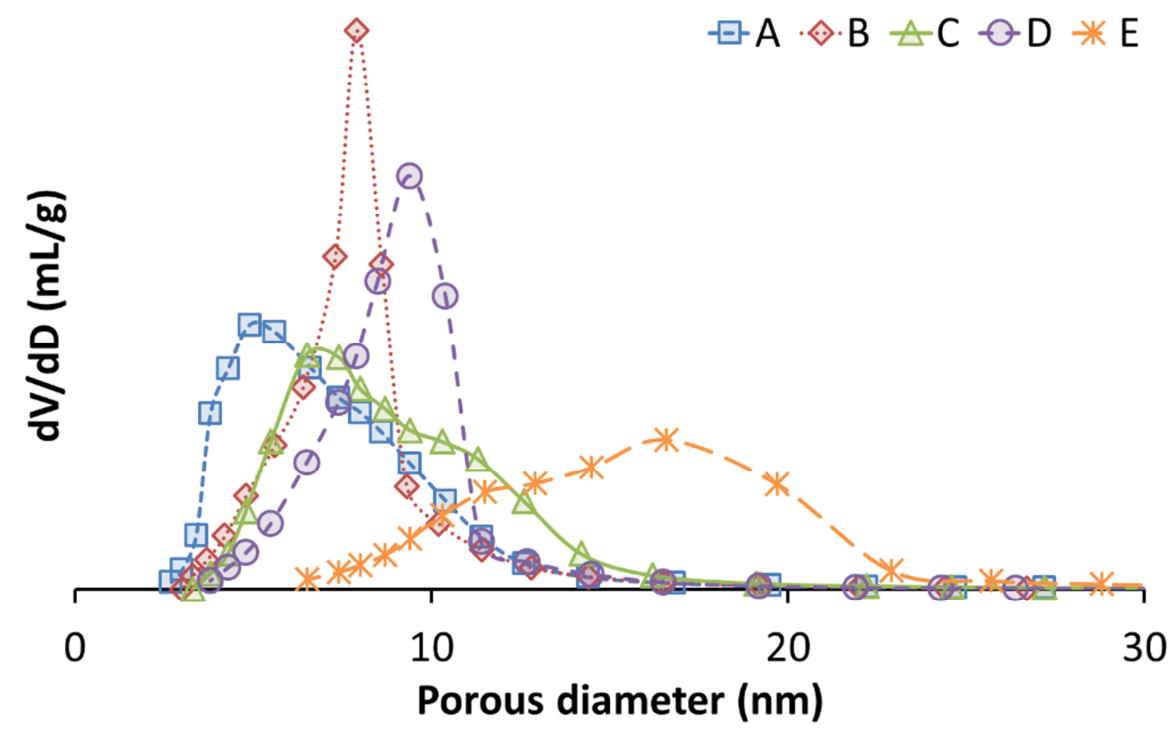

Figure 1. Alumina supports pore size distribution estimated by the BJH method 


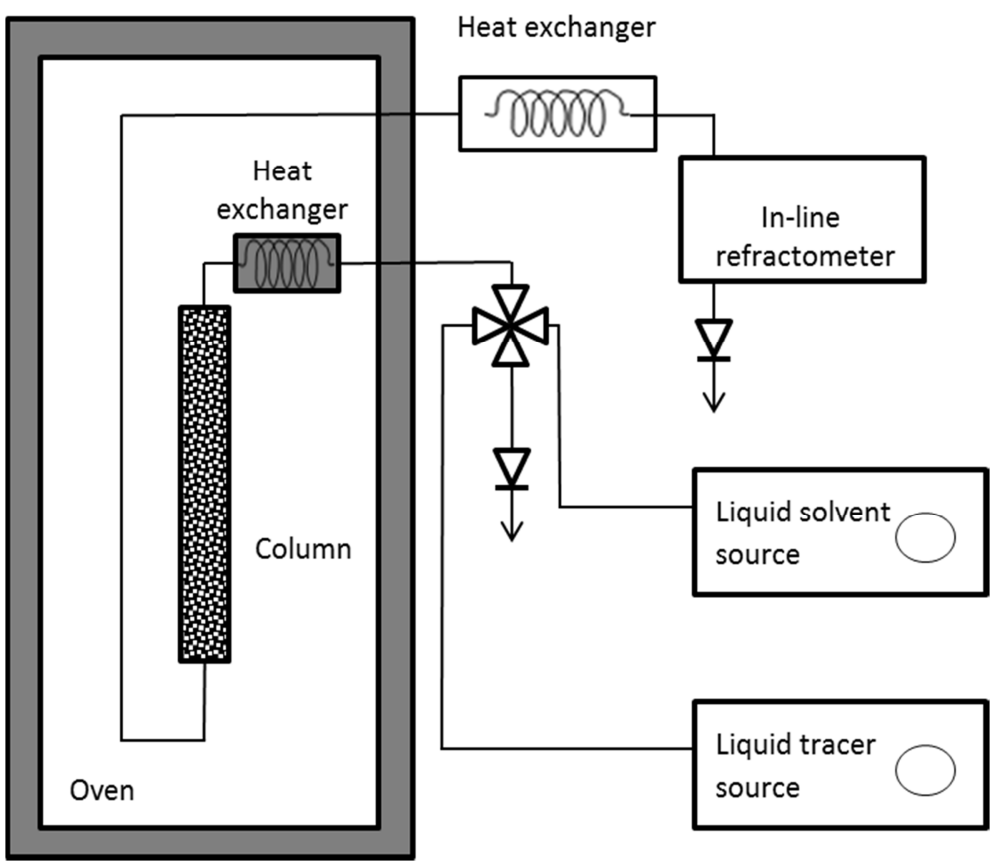

Figure 2. Inverse liquid chromatography setup
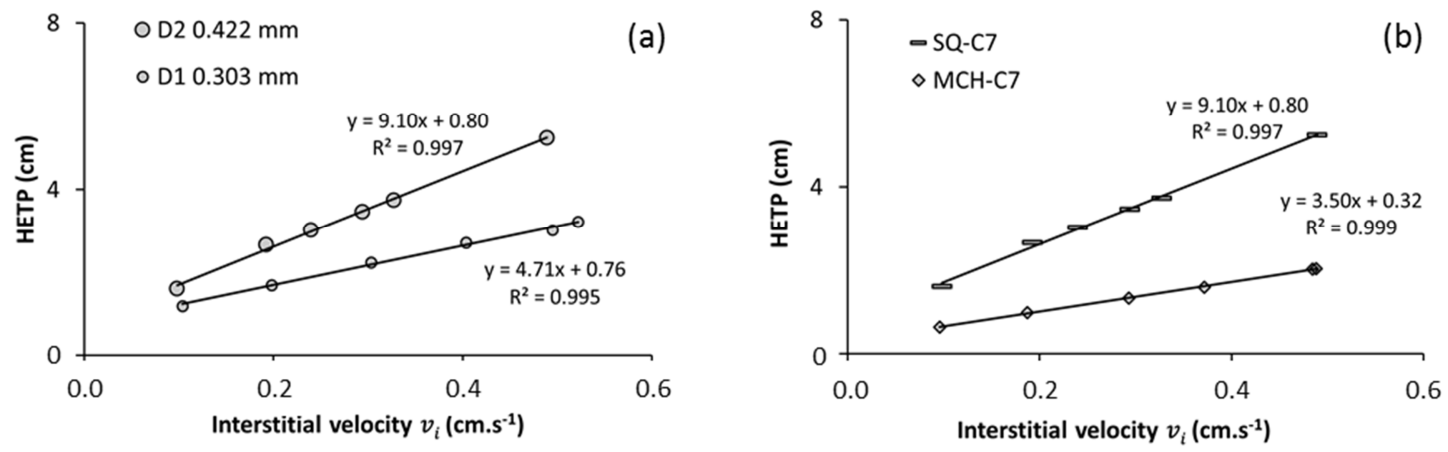

Figure 3. Van Deemter curves for alumina support D: (a) effect of particle size with SQ-C7 and (b) effect of the tracer size (MCH-C7 vs. SQ-C7) 


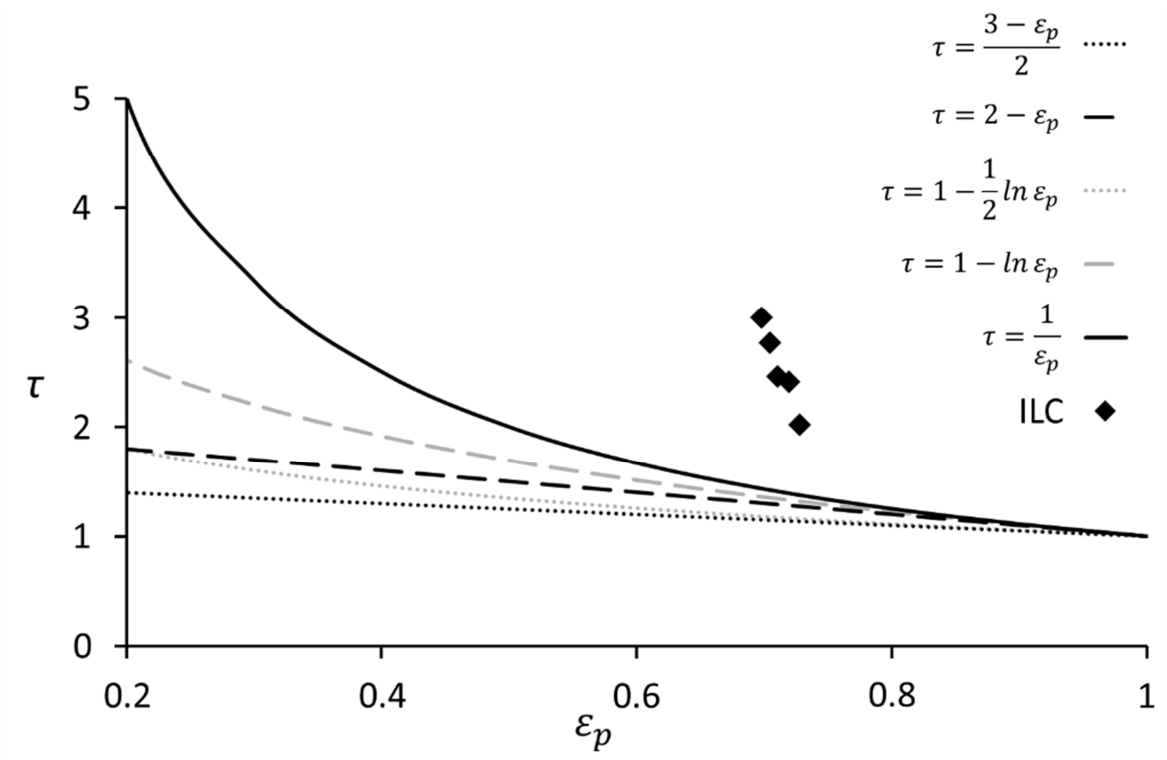

Figure 4. Theoretical and experimental (ILC) tortuosity-porosity values

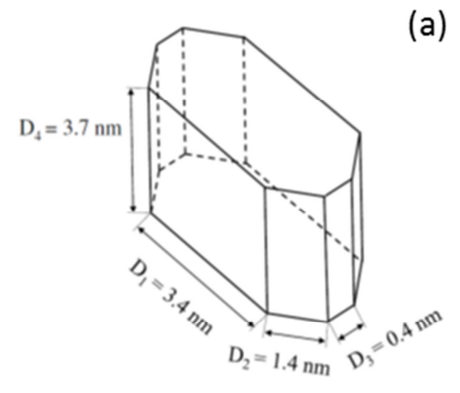

(a)

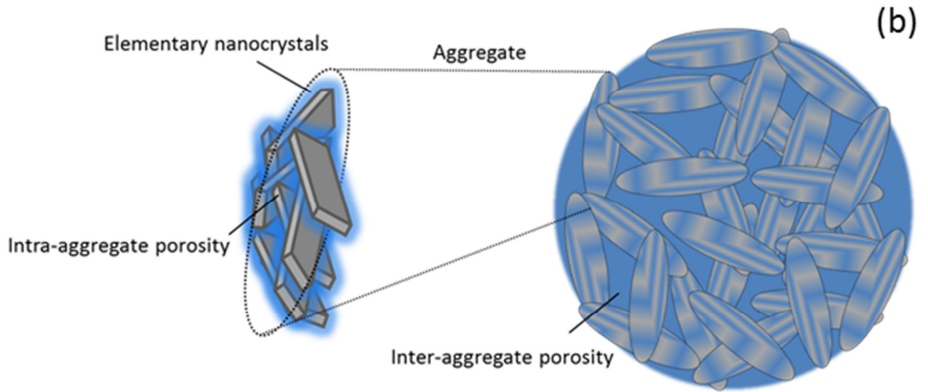

Figure 5. (a) Schematic representation of a typical alumina platelet shape ${ }^{[6]}$ and (b) Schematic representation of the two levels of porosity 

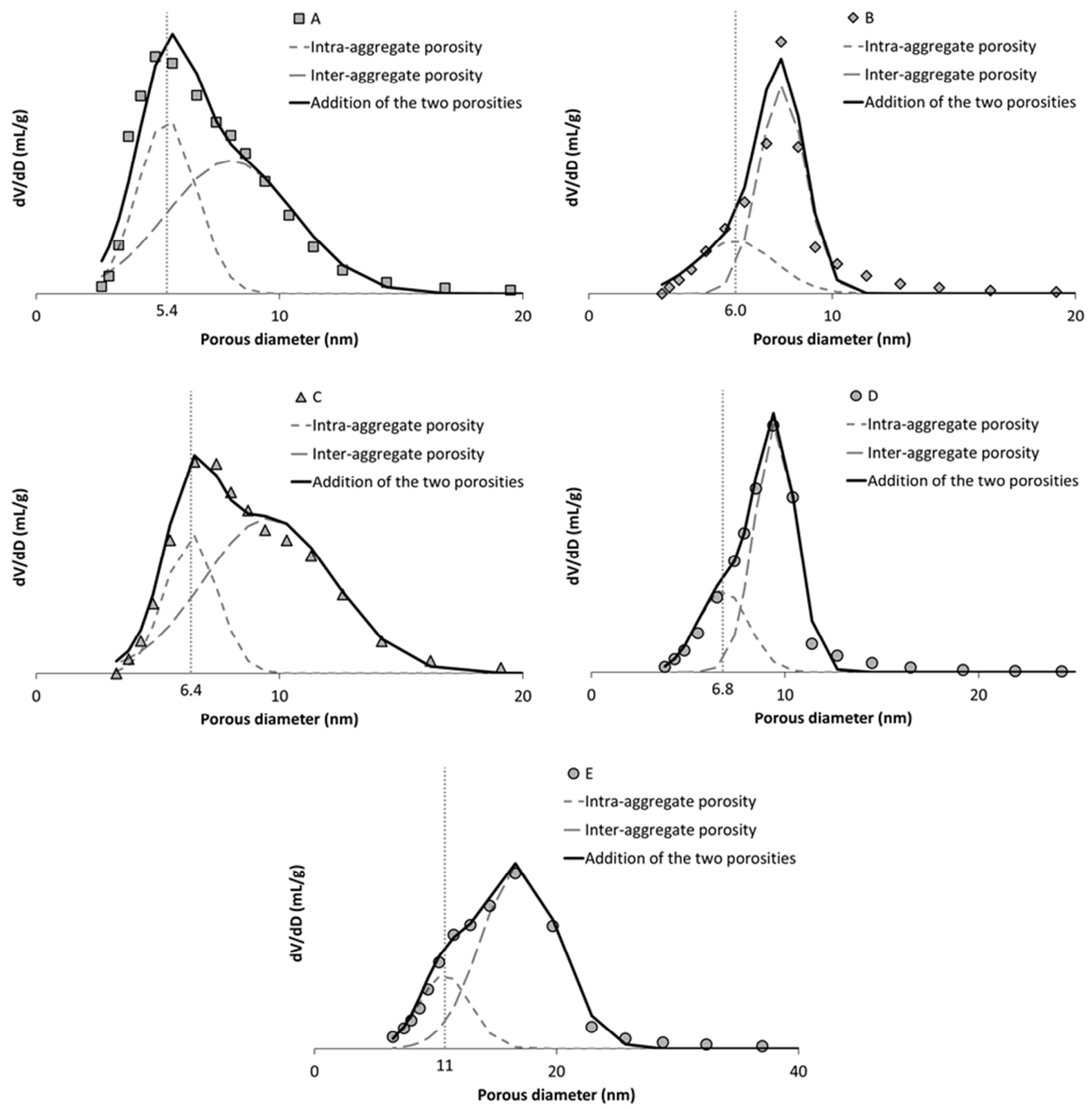

Figure 6. Deconvolution of the BJH pore size distributions for the various alumina supports into two pore size distributions, one for the porosity intra-aggregate and one for the porosity inter-aggregate. 


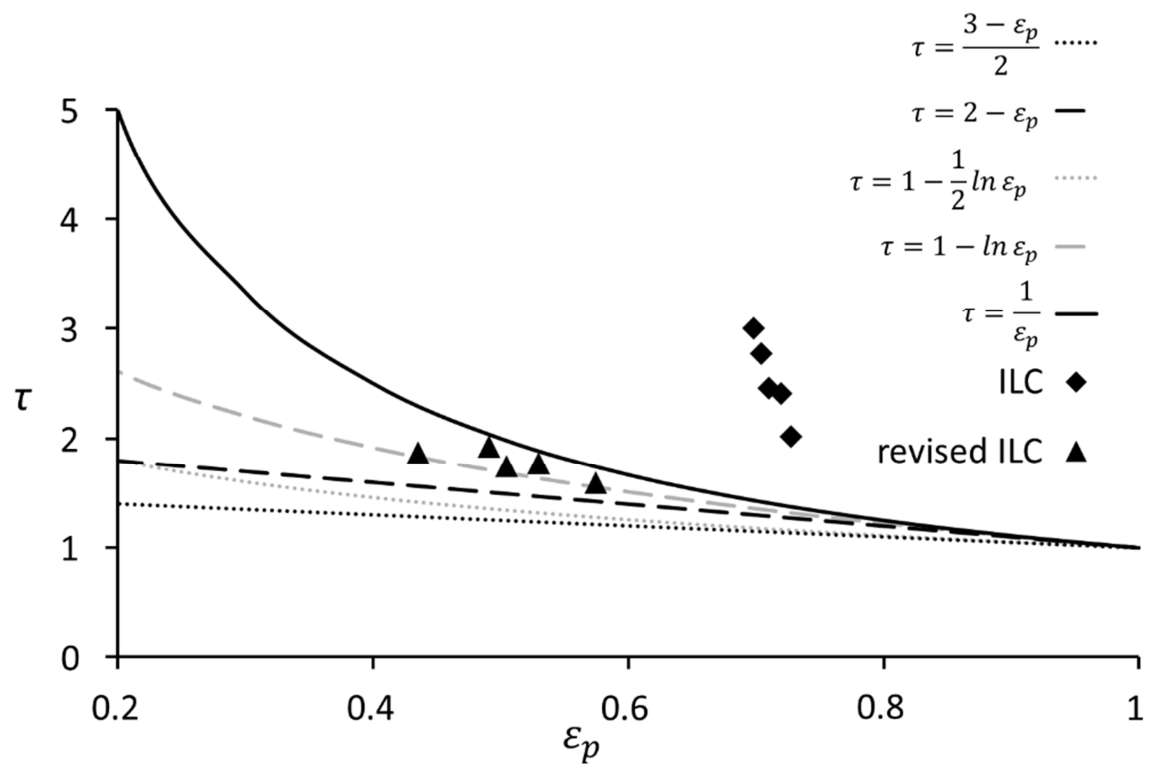

Figure 7. Theoretical and experimental (Inverse Liquid Chromatography) tortuosity-porosity relations. ILC corresponds to the uniform porosity representation - revised ILC corresponds to the two-level porosity representation. 